\title{
Zusammenhalts-Regionen - zur Theorie der Weltgesellschaft in der Sozialgeographie
}

\author{
Peter Dirksmeier ${ }^{1,2}$ and Angelina Göb ${ }^{1,2}$ \\ ${ }^{1}$ Institut für Wirtschafts- und Kulturgeographie, Leibniz Universität Hannover, \\ Scheiderberg 50, 30167 Hannover, Germany \\ ${ }^{2}$ Forschungsinstitut Gesellschaftlicher Zusammenhalt, Hannover, Germany \\ Correspondence: Peter Dirksmeier (dirksmeier@kusogeo.uni-hannover.de) and Angelina Göb \\ (goeb@kusogeo.uni-hannover.de)
}

Received: 16 April 2021 - Revised: 19 August 2021 - Accepted: 2 November 2021 - Published: 23 November 2021

Kurzfassung. The essay combines the concept of social cohesion with Rudolf Stichweh's system-theoretical concept of world society. These two approaches are joint hereafter with questions of spatial differentiation. The aim is to embed empirical micro-studies in macro-theoretical terms and to make them useful for empirical research in social geography. The construct of "cohesive region" demonstrates this by using the example of neighbourhoods.

\section{Problemstellung}

Die Theorie der Weltgesellschaft in der Lesart des Soziologen Rudolf Stichweh (2000, 2008) ,postuliert, dass es auf der Erde nur (noch) ein einziges Gesellschaftssystem gibt, das alle Kommunikationen und Handlungen in der Welt in sich einschließt" (Stichweh, 2017, 549). Gleichzeitig bedingt und ermöglicht Weltgesellschaft Diversität. Sie konstituiert sich als globaler sozialer Zusammenhang interner Differenzierung mit regionalen Varianzen (Stichweh, 2000). Die Betonung einer weltweiten Vernetzung und Interdependenz bietet für die empirisch arbeitende Sozialgeographie wichtige theoretische Bezugspunkte, Erklärungs- sowie Einordnungsmöglichkeiten ihrer empirischen Befunde. Sozialräumliche Prozesse in Nachbarschaften, wie z.B. Interaktionen, können so in einen Zusammenhang mit makrostrukturellen Entwicklungen gestellt und verstanden werden (Abb. 1). Lokale Phänomene, die mit Begriffen wie „Glokalisierung“ (Robertson, 1995), „Hyper-diversity“ (Kraftl et al., 2019) und „meaningful contact" (Valentine, 2008; Askins, 2016; Wilson, 2017) beschrieben und auf die Unterschiedlichkeit der jeweils involvierten lokalen Sozialitäten zurückgeführt werden, können aus weltgesellschaftlicher Perspektive als Bestandteil einer einzigen Gegenwartsgesellschaft aufgefasst werden.
Unser Beitrag zeigt im Folgenden das Potenzial der Theorie der Weltgesellschaft in der Lesart Rudolf Stichwehs für die Sozialgeographie am empirischen Forschungsproblem des gesellschaftlichen Zusammenhalts auf. Diesen definiert Rainer Forst zuvorderst als „ein übergreifendes Narrativ von sozialer Integration und Kooperation [...], das einem selbst und anderen eine relevante Zugehörigkeit zuschreibt und zusteht, auch wenn die anderen sonst anderen Vorstellungen und Praktiken folgen, etwa in der Differenz kultureller Lebensformen“ (2020, 50-51). Das Konstrukt des gesellschaftlichen Zusammenhalts in der Weltgesellschaft kann folglich nur einen Zusammenhalt Verschiedener beschreiben, der sozialräumlich variiert, gerade weil die Gesellschaft selbst gesellschaftsinterne Unterschiede (Un)gleichheit und (In)differenz - (re)produziert. Da die Theorie der Weltgesellschaft soziale Effekte der Globalisierung, Regionalisierung und Lokalisierung gleichermaßen integriert, kommt dem Ansatz Stichwehs ein hoher Stellenwert für die Begegnung aktueller Herausforderungen der modernen Gesellschaft zu. So erlaubt das grundlegende Konzept der Weltgesellschaft von einer gesellschaftlichen Einheit die Einbeziehung weltweit auftretender Fragen zur Akzeptanz von Diversität mit Anschlussmöglichkeiten für eine normative Ausrichtung des Begriffs als Konzeption (Forst, 2017; 2020) und damit für die Etablierung politisch-planerischer 


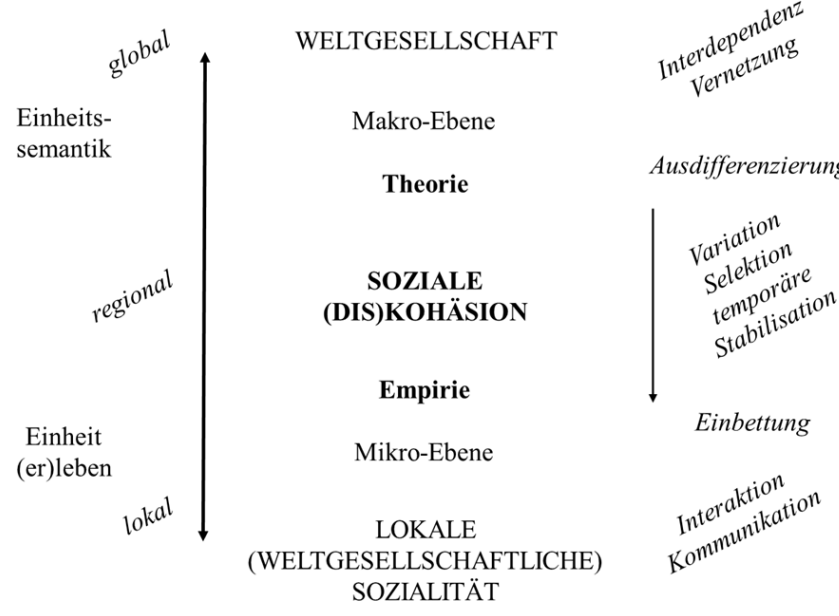

Abb. 1. Schematische Darstellung zum Zusammenhang von Weltgesellschaft und regionalen Gesellschaften in der Interdependenz von Mikro-Makro-Mechanismen (eigener Entwurf).

Umsetzungsstrategien. Zudem erweitert die makrotheoretische Einbettung kleinräumiger Untersuchungen in ein weltgesellschaftliches System - jenseits politisch-soziologischer Ordnungen (Meyer et al., 1997) - die Bandbreite möglicher Forschungsthemen in der Sozialgeographie sowie deren Theoriebildung.

\section{Interaktion und Gesellschaft - Zusammenhalten vs. Zusammenhalt}

Die Geographie der (Dis)kohäsion (Dirksmeier et al., 2020) zeigt im Rahmen der Forschung zum gesellschaftlichen Zusammenhalt auf, ,daß die wechselseitige Beeinflussung globaler und lokaler Zusammenhänge an der einzelnen Interaktion ablesbar sein muß" (Stichweh, 2000, 16). Daher setzen empirische Untersuchungen bei regional verortbaren Interaktionen an, um zu eruieren, ob und wie diese Interaktionsform(ierung)en gesellschaftlichen Zusammenhalt fördern, hemmen und/oder ,,neutralisieren“. Interaktionen können auf die Gesellschaft bezogen sein, stellen aber separate Sozialsysteme dar. Das Gesellschaftliche erscheint in diesen nur indirekt und partiell. So appräsentieren Interaktionen eine Selektion der einer Gesellschaft inhärenten Möglichkeiten bzw. Beschränkungen und repräsentieren Operationen in einer - als raumzeitlich definierte Episode - abgrenzbaren Einheit.

Mit Fokus auf die an der Interaktion beteiligten Gesellschaftsmitglieder zeigt sich, dass das Individuum sich in der Gegenwartsgesellschaft zumeist nur als Dividuum zeigen kann, d.h. als funktionaler Ausschnitt seiner Person. In unterschiedlichen Interaktionen mit unterschiedlichen Beteiligungsgraduierungen gelten dementsprechend unterschiedliche Rollen- und Verhaltenserwartungen, die an die Interaktionsteilnehmenden gestellt werden. Dies wird daran erkenn- bar, dass ein/e jede/r in jeder neuen oder veränderten Interaktion ein/e andere/r ist. Die unter diesen (selbst-)selektiven Bedingungen notwendigerweise ablaufende „Multiinklusion" (Nassehi, 2013, 35) in verschiedene Systeme bewirkt jedoch, dass Gesellschaftsmitglieder mit ihren diversen $\mathrm{Zu}-$ gehörigkeiten überwiegend segmentiert integriert sind. Obwohl nur wenige personenbezogene Aspekte dem Gegenüber in der jeweiligen Interaktion sichtbar oder zugänglich sind, können ausgeschlossene Aspekte (re)aktiviert werden und die Wahrscheinlichkeit von Abweichungen der Verhaltenserwartungen erhöhen. Mit zunehmender Komplexität innerhalb der Weltgesellschaft steigen Unsicherheiten und Uneindeutigkeiten im Umgang miteinander sowie Options- und Anschlussmöglichkeiten an das Verhalten anderer. Das Nichterwartbare wird zur Normalität, wenn alles und nichts passieren kann. Um auf diese Kontingenz zu reagieren, , gibt [es] offensichtlich eine Minimalmoral, die in einer basalen Akzeptanz aller anderen besteht, die sich diese nicht erst erarbeiten und verdienen müssen" (Stichweh, 2003a, 109) - aber eben auch nicht mehr als diese erwarten können.

Ein Schlüsselphänomen der modernen Gesellschaft ist nach Stichweh (2003a) die institutionalisierte Bekanntschaft, die auf einem Netzwerk von mehr oder weniger unpersönlichen, überwiegend funktionalisierten Bindungen aufruht. Diese meist flüchtigen sozialen Begegnungen basieren auf einem bewussten Verzicht auf ohnehin nur potenziell erhältlichen Informationen, die sich aus einer Fülle von Sinneseindrücken und medialen Inhalten zusammensetzen. In ihrer Individualität bzw. in ihrem Selektionsvermögen sind sich einander Begegnende so gleich, dass sie sich nicht mehr als Fremde wahrnehmen und bewerten können. Dies zeigt sich in einer Gleichbehandlung von Verschiedenem bzw. einer Nichtbeachtung und Thematisierung des Differenten (Stichweh, 2003a, 104). Bekanntschaft und Indifferenz fungieren somit als ,moderne Form von Sozialkapital“ (Stichweh, 2000, 224), mit der ein grundlegendes Vertrauen in andere - nicht als Fremde, sondern als Gleiche - einhergeht, das zu einer Reduktion von sozialer Komplexität beiträgt (Luhmann, 1989). Dieser Modus der Handlungsorientierung zeichnet sich durch einen riskanten Vorschuss auf ein künftiges Verhalten aus, das nur auf der Grundlage einer bewussten Entscheidung von Differenz-Negationen umgesetzt werden kann. Indifferenz charakterisiert also eine Einstellung, die sich in einem gleichwertigen Nebeneinander manifestiert und erst bei Differenzkonstruktion - einer Abgrenzung durch (eigene und fremde) Zugehörigkeitszuschreibungen - Dualismen aufbaut, die (dis)kohäsiv wirksam werden. Das Aufeinandertreffen von Interagierenden kann sich dementsprechend positiv-konstruktiv, negativ-destruktiv oder neutraldistanziert entwickeln. Es führt zu einem Mit-, Gegen- oder Nebeneinander der Beteiligten, das mehr oder weniger konflikthaft ist und eine kontextualisierte Aushandlung von Normen erforderlich macht.

In solchen interaktiven Bestimmungsprozessen kommt Wir- und Ihr-Identifikationen, d.h. der Grup- 
pen(re)etablierung bzw. -ablehnung, eine herausgehobene Bedeutung für die Selbstkategorisierung und Kollektivierung zu. Identifikationen von und mit Anderen und Anderem verbinden und trennen zugleich und schaffen darüber hinaus Orientierung, Sicherheit und Vertrautheit. Diese Zuund Einordnungen befördern das Erwarten-Können, das in sozial fungierenden Einheitssemantiken evident wird. Dabei entzünden sich diese auf Geschlossenheit beruhenden Selbstbeschreibungen von Gruppen, Gemeinschaften bzw. lokalen Sozialitäten an der Erfahrung von Differenz. Aufgrund der Ausdifferenzierung von Sozialsystemen, d.h. der Differenzierung innerhalb und zwischen lokalen Sozialitäten, existieren in der Weltgesellschaft jedoch mannigfaltige Einheitskonzepte parallel - ohne miteinander in Einklang stehen zu müssen. So emergieren im Rahmen der Selbstgestaltungsmöglichkeiten von lokalen Gruppen vielfältige „,(Welt)gemeinschaften“ (Stichweh, 2017, 558), die weniger durch enge Verknüpfungen als durch wechselseitige Beobachtung bestehen und zusammengehalten werden. Zusammenhalt konstituiert sich in der Weltgesellschaft dementsprechend als Zusammenhalt Verschiedener. Insofern stellt sich die Frage, ob und wie sich eine weltgesellschaftliche Einheit bei regionaler und sozialer Varianz „zusammenhaltskonform“ verhalten kann, will oder muss, z.B. im Rahmen von Inszenierungen geplanter Weltereignisse etwa im Sport (z.B. Olympische Spiele, Fußball-WM) (Stichweh, 2003b) oder bei natürlichen Geschehnissen und Katastrophen wie der Corona-Pandemie. Andersherum kann gefragt werden, welche empirisch nachweisbaren Zusammenhaltskonstellationen wo und wie (lange) in und zwischen welchen Systemen auftreten müssen, um dem gegenwärtig vorherrschenden Diskurs einer sich-selbst-spaltenden Gesellschaft zu entgegnen. In der Aufeinanderbezogenheit der sozialen Systeme Gesellschaft und Interaktion fällt auf, dass sich einheitsproklamierende Semantiken fast ausschließlich auf den Zusammenhalt der Gesellschaft als Ganzes beziehen, weniger auf die regionalen Spezifika und ihre Aushandlungspraktiken (Arant, Larsen and Boehnke, 2016). Ge- und erlebter Zusammenhalt vor Ort (im Sinne eines regionalen resp. gesellschaftlichen Zusammenhaltens bzw. Zusammenhalt-Machens) bedarf offensichtlich weniger eines artikulierten Narratives, wenn Komplexität reduziert, sozialräumliche Überschaubarkeit im täglichen Interagieren gegeben ist.

\section{Regionalisierung von Zusammenhalt - die Zusammenhalts-Region}

Die Verschiedenheit in der Weltgesellschaft ermöglicht die Konstruktion verschiedener Zusammenhaltsvorstellungen und bringt eine „Diversität von Möglichkeiten“ (Stichweh, 2000, 214) hervor. Unter der Prämisse, dass sich Weltgesellschaft Diversität als Variations- und Selektionsspielraum produziert und erhält, muss gesellschaftlicher Zusam- menhalt auch regional ausdifferenziert in Erscheinung treten. Unser Blick auf Diversität, also den Erhalt von sachlicher und sozialer Differenz im zeitlichen Verlauf, wird erweitert um eine regionale Verortung von Varianz in Hinblick auf die Frage: Wie stellt sich Zusammenhalt in Interaktionen für wen, wann und wo, insbesondere räumlich situiert, dar? Auf diesem Gedankengang baut die „Theorie der Regionalisierung auf, d.h. eine Theorie der neu-entstehenden regionalen und lokalen Differenzen im System der Weltgesellschaft" (Stichweh, 2000, 125). Regionalisierung beschreibt demzufolge eine ,Form der internen Differenzierung der modernen Gesellschaft" (Stichweh, 2000, 217). Diese Differenzierung bringt regionale Varianzen in sozialen Strukturen hervor. Regionen nehmen dementsprechend unterschiedliche Formen (wie Grenzen) und Formierungen (wie Funktionskopplungen und Ausprägungsgraduierungen) in verschiedenen Weltgegenden an und ordnen regional voneinander abweichende Phänomene. Sie repräsentieren einen Status quo, denn sie wirken wie ,evolutionäre Nischen“ (Stichweh, 2000, 217) und transformieren sich im Prozess der Regionalisierung durch Mechanismen der Variation, Selektion und relativen Stabilisation. Damit lassen sie sich als spezifische Zusammenhalts-Regionen konstruieren und für die sozialgeographische Forschung als Orte (dis)kohäsiver (Kommunikations-)Praktiken untersuchen.

Zusammenhalts-Regionen sollen - nach unserem Vorschlag - empirische Einheiten zur räumlichen Ordnung von (dis)kohäsiven Interaktionen genannt werden. In diesen interagieren unterschiedliche soziale Gruppen in einem ZeitRaum mit-, gegen- oder nebeneinander und bilden dabei unterschiedliche Formen von Zusammenhalt aus (Dirksmeier et al., 2020), die es zu kontextualisieren und kategorisieren gilt. Regional verortbare Interaktionsepisoden sind offen für Transformationen und dadurch eine ,wahrscheinliche Quelle von Diversität und Perzeption von Ungleichheit in der Weltgesellschaft" (Stichweh, 2000, 218). Als solche können sie emergente Zusammenhaltsordnungen (immer wieder neu) hervorbringen, aber auch stabilisieren (Abb. 2).

Am Beispiel „Nachbarschaft“" soll im Folgenden eine Zusammenhalts-Region betrachtet werden, die als soziale wie räumliche (An)ordnung von Gesellschaft (Hamm, 2000, 173) definierbar ist und als Rahmen für die Untersuchung von Zusammenhaltskonstitutionen herangezogen werden kann. In Nachbarschaften und durch die Praxis des Nachbarschaftens kann Zusammenhalt unmittelbar generiert und perpetuiert werden - in und zwischen sozialen Gruppen wie räumlichen Einheiten als intra- und internachbarschaftlicher Zusammenhalt. Als alltäglicher Gebrauchs- und Erfahrungsraum sind sie bedeutsam, da sie Vertrautheit mittels sozialräumlicher Überschaubarkeit offerieren: Mensch kann sich in Nachbarschaften problemlos orientieren, kennt sich und weiß gemeinhin, wie mensch sich zu verhalten hat (Valentine and Harris, 2016). Selbst wenn soziale Identifikation und raumbezogene Zugehörigkeitszuschreibungen ausbleiben, z.B. als „absent ties“ (Blokland and Nast, 2014) oder 


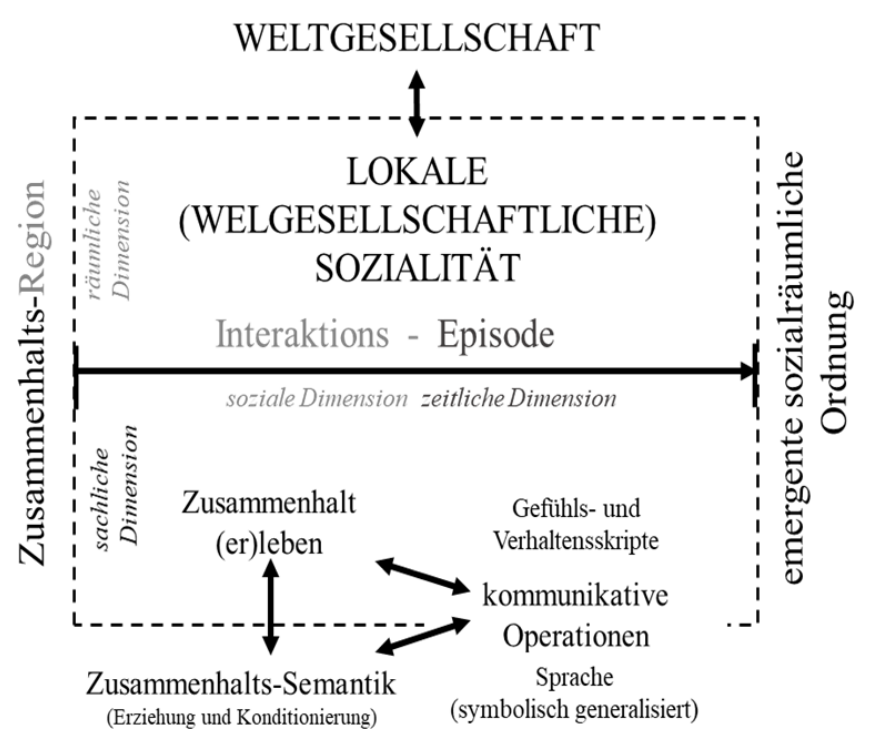

Abb. 2. Schematische Darstellung zur Analyse von Zusammenhalts-Regionen anhand von Interaktionsepisoden (eigener Entwurf).

„weak ties“ (Granovetter, 1973), bestehen ordinäre Kontaktmöglichkeiten mit der Potenzialität, bedeutungsvoll zu werden und ein „meeting“ in ein „mating“ (Small and Adler, 2019, 117) verwandeln zu können. Dieser tägliche Kontakt kann neben solidarischem Miteinander aber auch Vorurteile und Konflikte erzeugen oder unverbindlich und minimalsympathisch verbleiben. Vor dem Hintergrund einer Ausbildung von super-diversen Nachbarschaften (Vertovec, 2007) ist ein Zusammentreffen unterschiedlicher Einstellungs- und Verhaltensweisen von Bewohner:innen zu erwarten, die Ungewissheit und Unsicherheit im direkten Kontakt wahrscheinlicher machen. Empirische Studien belegen in diesem $\mathrm{Zu}-$ sammenhang ambivalente Entwicklungen und Konsequenzen: So schlägt sich eine steigende ethnische Diversität in positiven, negativen und/oder neutralen Kohäsionsmustern nieder (Sturgis et al., 2014; van der Meer and Tolsma, 2014; Koopmans and Schaeffer, 2016). Daher gelten Nachbarschaften nach wie vor als „Räume des Widerspruchs - Orte der Geselligkeit und des Konflikts“ (Phillips et al., 2014, 55), der Minimierung und Maximierung von Differenz, Inklusion und Exklusion. In Anbetracht dessen scheint die Etablierung von Nachbarschafts-Normen als verortbaren Verhaltenskodizes bzw. die Aushandlung von Routinen und Regeln im Umgang mit Andersheit notwendig - auch oder gerade im Konflikt verschiedener Akteur:innen. Welche Kohäsionsmuster sich in welchen räumlichen Handlungskontexten von Nachbarschaft ausbilden, wie dies gelingt bzw. misslingt, ist weitestgehend unbekannt. Des Weiteren ist unklar, welche Effekte die Nachbarschaft selbst (Galster, 2012; Kwan, 2018; Petrović et al., 2020) - in Hinblick auf Ausstattung, Verteilung, Zugang zu Infrastrukturen, Einrichtungen und (Umwelt-)Ressourcen usw. - auf die Genese von $\mathrm{Zu}$ - sammenhalt hat. Da die meisten Menschen in räumlich deutlich ausgreifenderen Zusammenhängen als in ihrer Nachbarschaft leben, sind Nachbarschaftseffekte auch unter Berücksichtigung der zeitlichen Dimension zu betrachten. Offensichtlich ist, dass die Wohndauer, Gewöhnungs- und Anpassungseffekte vor Ort, aber auch globalisierte bzw. digitalisierte Aktionsräume und (Freundschafts-)Netzwerke, die Bewohner:innen zum Teil weniger ,place-based als place-less“ (Farahani, 2016, 2) (inter)agieren lässt. Daneben sind es die Geschichte und Traditionen des Ortes, (Leit-)Bilder sowie politisch-institutionelle Strukturen und Systeme, die den $\mathrm{Zu}-$ sammenhalt - positiv wie negativ - rahmen (Valentine and Sadgrove, 2012). Während sich sozialstrukturelle Heterogenität zwischen Nachbarschaften positiv auf den Zusammenhalt auswirkt, stehen Unterschiede innerhalb von Nachbarschaften sozial kohäsiven Prozessen entgegen (Sturgis et al., 2014; Wickes et al., 2019). Entscheidend in diesem Zusammenhang scheint die räumliche Verteilung sowie das Ausmaß der sozialen und ethnischen Durch- bzw. Entmischung zu sein.

\section{Ausblick: Regionalisierung von Zusammenhalt in der Weltgesellschaft}

Die Analyseeinheit der Zusammenhalts-Region eröffnet der Sozialgeographie, wie hier am Beispiel der nachbarschaftlichen Interaktion aufgezeigt, einen empirischen Zugang zu der Frage, warum Menschen sich möglicherweise häufiger gegen Indifferenz im Umgang mit Differenz und stattdessen für die aktive Ausgrenzung, Fremdheitszuweisung und damit konflikthafte Auseinandersetzungen entscheiden. Armin Nassehi folgert dementsprechend, dass ,,den besten Zusammenhalt wohl eine Gesellschaft [bietet], die auf eine gepflegte, voraussetzungsvolle und bekenntnisfähige Form des $\mathrm{Zu}$ sammenhalts verzichten kann" $(2013,44)$. Einen allgemeingültigen Zusammenhalt scheint es vor dem Hintergrund einer Wertevielfalt innerhalb und zwischen verschiedensten regionalen Gruppen resp. lokalen Sozialitäten ohnehin kaum geben zu können. Die neuerliche Konjunktur der nur schwer definitorisch fassbaren Idee des gesellschaftlichen Zusammenhalts deutet daher auf eine empirische Krise der für die Weltgesellschaft basalen Annahme von Gleichbehandlung von Verschiedenem und Verschiedenen hin, die nicht ohne Weiteres als inhärent vorausgesetzt werden kann.

Unter Bedingungen einer funktional differenzierten Gesellschaft erwecken aktuelle Medienberichte den Eindruck, dass das Bedürfnis nach Einheitssemantiken steigt. Der Begriff des gesellschaftlichen Zusammenhalts wird meist dann herangezogen, wenn übergeordnete gemeinsame Ziele zu fehlen scheinen, sozialräumliche Überschaubarkeit zu sinken sowie ein Verlust der Vereinbarkeiten von Systemeinheiten und Anschlussmöglichkeiten droht. Um der Realität moderner Gesellschaften gerecht(er) zu werden, plädiert Rainer Forst deshalb dafür, ,,[dass] man Zusammenhalt als eine 
Form der Integration denken [sollte], die die Pluralität individueller Gruppenzugehörigkeiten und systemischer Ausdifferenzierungen reflektiert und soziale wie auch politische Prozesse der Kooperation vorsieht, die Konflikte einschlieBen“ (2020, 44-45). Dabei wirken weniger die Konflikte eo ipso integrativ und assoziativ, sondern deren Austragungsarten, die intersubjektives Lernen und Reflektieren über Differenzen ermöglichen und Zusammenhalt stiften können.

Da Weltgesellschaft selbst Diversität wie Homogenität gleichzeitig und überall produziert, sollte dem Betrachtungsmaßstab der Interaktion bei der empirischen Forschung eine besondere Aufmerksamkeit zugesprochen werden. So bieten Nachbarschaften kleinräumig die Möglichkeit einer intensiven, die Gesellschaftsmitglieder integrierenden Analyse der Variationsbedingungen und -gründe von gesellschaftlichem Zusammenhalt in verschiedenen (Welt-)Gegenden. Die Arbeiten zur Theorie der Weltgesellschaft von Rudolf Stichweh leisten hier für die Sozialgeographie eine wichtige konzeptionelle Grundlage. Sie rahmen die flottierenden politischen wie wissenschaftlichen Diskurse um gesellschaftliche Spaltung und fehlenden sozialen Zusammenhalt. Darüber hinaus bieten sie einen theoretischen Ausgangspunkt für die Sozialgeographie, um mögliche Forschungsdesiderate im Zusammenhang von Zusammenhalt und Interaktion aufzuzeigen. Für die sozialgeographische Theorie der Regionalisierung ist die Erklärung von solcherart weltgesellschaftlich gerahmten Aspekten, Fragen und Desideraten des sozialen Zusammenhalts, insbesondere auf lokaler Ebene, eine bis dato neue und bedeutende Forschungsaufgabe.

Datenverfügbarkeit. Für diesen Artikel wurden keine Datensätze genutzt.

Autorenmitwirkung. Beide Autor*innen haben den Artikel gemeinsam konzipiert und verfasst.

Interessenkonflikt. Die Autor*innen erklären, dass kein Interessenkonflikt besteht.

Haftungsausschluss. Copernicus Publications bleibt in Bezug auf gerichtliche Ansprüche in veröffentlichten Karten und institutionellen Zugehörigkeiten neutral.

Finanzierung. This research has been supported by the Bundesministerium für Bildung und Forschung (grant no. 01UG2050FY).

Begutachtung. This paper was edited by Benedikt Korf and reviewed by one anonymous referee.

\section{Literatur}

Arant, R., Larsen, M., and Boehnke, K.: Sozialer Zusammenhalt in Bremen, Bertelsmann Stiftung, Gütersloh, 2016.

Askins, K.: Emotional citizenry: everyday geographies of befriending, belonging and intercultural encounter, T. I. Brit. Geogr. N. S., 41, 515-527, https://doi.org/10.1111/tran.12135, 2016.

Blokland, T. and Nast, J.: From public familiarity to comfort zone: the relevance of absent ties for belonging in Berlin's mixed neighborhoods, Int. J. Urban Regional, 38, 1142-1159, https://doi.org/10.1111/1468-2427.12126, 2014.

Dirksmeier, P., Göb, A., Herrmann, S., Ibendorf, J., Knaps, F., Othengrafen, F., and Ruffing, E.: Räumliche Unterschiede und gesellschaftlicher Zusammenhalt, in: Gesellschaftlicher Zusammenhalt. Ein interdisziplinärer Dialog, edited by: Deitelhoff, N., Groh-Samberg, O., and Middell, M., Campus, Frankfurt, New York, 273-294, 2020.

Farahani, L. M.: The value of the sense of community and neighbouring, Housing, Theory and Society, 33, 357-376, https://doi.org/10.1080/14036096.2016.1155480, 2016.

Forst, R.: Toleranz im Konflikt. Geschichte, Gehalt und Gegenwart eines umstrittenen Begriffs, Suhrkamp, Frankfurt/M., 2017.

Forst, R.: Gesellschaftlicher Zusammenhalt. Zur Analyse eines sperrigen Begriffs, in: Gesellschaftlicher Zusammenhalt. Ein interdisziplinärer Dialog, edited by: Deitelhoff, N., Groh-Samberg, O., and Middell, M., Campus, Frankfurt, New York, 41-53, 2020.

Galster, G.C.: The mechanism(s) of neighbourhood effects: theory, evidence, and policy implications, in: Neighbourhood Effects Research: New Perspectives, edited by: Van Ham M., Manley, D., Bailey, N., and Simpson Land Maclennan, D., Springer, New York, 23-56, 2012.

Granovetter, M. S.: The strength of weak ties, Am J. Sociol., 78 , 1360-1380, https://doi.org/10.1086/225469, 1973.

Hamm, B.: Nachbarschaft, in: Großstadt: Soziologische Stichworte, edited by: Häußermann, H., Springer, Wiesbaden, 173-182, 2000.

Koopmans, R. and Schaeffer, M.: Statistical and perceived diversity and their impacts on neighborhood social cohesion in Germany, France and the Netherlands, Soc. Indic. Res. 125, 853-883, https://doi.org/10.1007/s11205-015-0863-3, 2016.

Kraftl, P., Bolt, G., and Van Kempen, R.: Hyper-diversity in/and geographies of childhood and youth, Soc. Cult. Geogr., 20, 1189-1197, https://doi.org/10.1080/14649365.2018.1491619, 2019.

Kwan, M.-P.: The limits of the neighborhood effect: contextual uncertainties in geographic, environmental health, and social science research, Ann. Am. Assoc. Geogr., 108, 1482-1490, https://doi.org/10.1080/24694452.2018.1453777, 2018.

Luhmann, N.: Vertrauen. Ein Mechanismus der Reduktion sozialer Komplexität, Enke, Stuttgart, 1989.

Meyer, J. W., Boli, J., Thomas, G. M., and Ramirez, F. O.: World Society and the nation-state, Am J. Sociol., 103, 144-181, https://doi.org/10.1086/231174, 1997.

Nassehi, A.: Inklusion, Exklusion, Zusammenhalt. Soziologische Perspektiven auf eine allzu erwartbare Diagnose, in: Was hält Gesellschaften zusammen? Der gefährdete Umgang mit Pluralität, edited by: Reder, M., Pfeifer H., and Cojocaru, M.-D., Kohlhammer, Stuttgart, 31-46, 2013. 
Petrović, A.; Manley, D., and van Ham, M.: Freedom from the tyranny of neighbourhood: rethinking sociospatial context effects, Prog. Hum. Geog., 44, 1103-1123, https://doi.org/10.1177/0309132519868767, 2019.

Phillips, D., Athwal, B., Robinson, D., and Harrison, M.: Towards intercultural engagement: building shared visions of neighbourhood and community in an era of new migration, J. Ethn. Migr. Stud., 40, 42-59, https://doi.org/10.1080/1369183X.2013.782146, 2014.

Robertson, R.: Glocalization: time-space and homogeneityheterogeneity, in: Global Modernities, edited by: Featherstone, M., Lash, S., and Robertson, R., Sage, London, 25-44, 1995.

Small, M. L. and Adler, L.: The role of space in the formation of social ties, Annu. Rev. Sociol., 45, 111-132, https://doi.org/10.1146/annurev-soc-073018-022707, 2019.

Stichweh, R.: Die Weltgesellschaft. Soziologische Analysen, Suhrkamp, Frankfurt am Main, 2000.

Stichweh, R.: Fremdheit in der Weltgesellschaft - Indifferenz und Minimalsympathie, in: Science + Fiction. Zwischen Nanowelt und globaler Kultur, edited by: Iglhaut, S. und Spring, T., Jovis, Berlin, 98-110, 2003a.

Stichweh, R.: Der 11. September 2001 und seine Folgen für die Entwicklung der Weltgesellschaft, in: Asymmetrische Konflikte und Terrorismusbekämpfung. Prototypen zukünftiger Kriege? edited by: Collmer, S. und Kümmel, G., Nomos, Baden-Baden, 7-16, 2003b.

Stichweh, R.: Das Konzept der Weltgesellschaft: Genese und Strukturbildung eines globalen Gesellschaftssystems, Rechtstheorie, 39, 329-355, 2008.
Stichweh, R.: Weltgesellschaft, in: Bonner Enzyklopädie der Globalität, edited by: Künhardt, L. and Mayer, T., Springer, Wiesbaden, 549-560, 2017.

Sturgis, P.; Brunton-Smith, I., Kuha, J., and Jackson, J.: Ethnic diversity, segregation and the social cohesion of neighbourhoods in London, Ethnic Racial Stud., 37, 1286-1309, https://doi.org/10.1080/01419870.2013.831932, 2014.

Valentine, G.: Living with difference: reflections on geographies of encounter, Prog. Hum. Geog., 32, 323-337, https://doi.org/10.1177/0309133308089372, 2008.

Valentine, G. and Harris, D.: Encounters and (in)tolerance: perceptions of legality and the regulation of space, Soc. Cult. Geogr., 17, 913-932, https://doi.org/10.1080/14649365.2016.1139171, 2016.

Valentine, G. and Sadgrove, J.: Lived difference: a narrative account of spatiotemporal processes of social differentiation, Environ. Plann. A, 44, 2049-2063, doi.org/10.1068/a44491, 2012.

van der Meer, T. and Tolsma, J.: Ethnic diversity and its effects on social cohesion, Annu. Rev. Sociol., 40, 459-478, https://doi.org/10.1146/annurev-soc-071913-043309, 2014.

Vertovec, S.: Super-diversity and its implications, Ethnic Racial Stud., 30, 1024-1054, https://doi.org/10.1080/01419870701599465, 2007.

Wickes, R., Zahnow, R., Corcoran, J., and Hipp, J. R.: Neighbourhood social conduits and resident social cohesion, Urban Stud., 56, 226-248, https://doi.org/10.1177/0042098018780617, 2019.

Wilson, H.: On geography and encounter: bodies, borders, and difference, Prog. Hum. Geog., 41, 451-471, https://doi.org/10.1177/0309132516645958, 2017. 